\title{
Complex earthquake sequences on simple faults
}

\author{
C. Cattania ${ }^{1}$ \\ ${ }^{1}$ Department of Geophysics, Stanford University, Stanford, CA
}

\section{Key Points:}

- Numerical simulations and fracture mechanics predict the occurrence of partial ruptures on sufficiently large faults loaded by creep

- Earthquake statistics is controlled by the ratio of fault dimension to a critical length related to the nucleation dimension

- Large faults exhibit afterslip driven temporal clustering and power-law distribution of seismic moments with a theoretical b-balue of $3 / 4$ 


\section{Abstract}

While power-law distributions in seismic moment and inter-event times are ubiquitous in regional earthquake catalogs, the statistics of individual faults remains controversial. Continuum fault models without heterogeneity typically produce characteristic earthquakes or a narrow range of sizes, leading to the view that regional statistics originate from interaction of multiple faults. I present theoretical arguments and numerical simulations demonstrating that seismicity on homogeneous planar faults can span several orders of magnitude in rupture dimensions and inter-event times, if the fault dimension $W$ is sufficiently large compared to a characteristic length $L_{c r i t}$, related to the nucleation dimension. Large faults are increasingly less characteristic, with the fraction of systemsize ruptures proportional to $\left(L_{c r i t} / W\right)^{1 / 2}$. Earthquake statistics for large $W / L_{\text {crit }}$ is remarkably close to nature, exhibiting Omori decay and power-law distributed rupture lengths. Simple crack models are consistent with a Gutenberg-Richter distribution with $b=3 / 4$, and provide a physical basis for these distributions on individual faults.

\section{Introduction}

Seismic hazard models are one of the most societally relevant products of earthquake research, but they often rely on poorly tested assumptions due to the scarcity of data on the recurrence interval of large earthquakes. Whether individual faults are more characteristic than predicted by the Gutenberg-Richter distribution remains a subject of discussion (Parsons \& Geist, 2009; Schwartz, 2010; Page, 2010; Parsons et al., 2012; Kagan et al., 2012; Page \& Felzer, 2015; Mulargia et al., 2017; Parsons et al., 2018; Stirling \& Gerstenberger, 2018).

The debate on the frequency-size distribution of earthquakes is echoed in the earthquake physics community. Early earthquake cycle simulations of planar faults in an elastic medium produced sequences of periodic, system-size events (Tse \& Rice, 1986; Rice, 1993). Subsequent studies found that sub-system-size events (partial ruptures) occur for a small enough slip weakening distance (Lapusta et al., 2000; Lapusta, 2003), or equivalently for a sufficiently large fault dimension (Wu \& Chen, 2014; Erickson et al., 2011; Herrendorfer et al., 2015; Werner \& Rubin, 2013; Cattania \& Segall, 2019). While simple limit cycles with few or no partial ruptures are typically produced by correctly discretized models, under resolved simulations exhibit richer slip complexity, including a power-law distribution of rupture dimensions (Rice, 1993; Lapusta et al., 2000; Ben-Zion 
\& Rice, 1995). Even though these models do not correctly solve the continuum equations, one interpretation is that oversized cells represent distinct fault segments, and can be considered a proxy for geometrical heterogeneity (Ben-Zion \& Rice, 1995). Similarly, discrete models of faults (such as cellular automata (Bak \& Tang, 1989; Olami et al., 1992) or discrete elastic models (Burridge \& Knopoff, 1967)) produce a power-law distribution of earthquake sizes, analogous to what is observed in nature.

The discrepancy between the predominantly characteristic and periodic behavior of continuum models and the rich complexity of discrete models led to the view that the statistics of seismicity on a regional scale is controlled by the discrete nature of faults (BenZion \& Rice, 1995; Ben-Zion, 2008) or by frictional/geometrical fault heterogeneity (e.g. Hillers et al. (2007); Aochi and Ide (2009); Kaneko et al. (2010); Dublanchet et al. (2013)); the latter is also understood to be responsible for partial ruptures on megathrust faults $(\mathrm{Li}$ et al., 2018; Qiu et al., 2016; Dal Zilio et al., 2019). An important question is then: if fault roughness, segmentation and the interaction between separate fault segments are responsible for earthquake statistics on a regional scale, are relatively smooth, isolated faults more likely to exhibit characteristic quasi-periodic behavior?

Here I address this question from a fracture mechanics perspective. From simple energy balance arguments, I demonstrate that an homogeneous velocity-weakening (VW; stick-slip) fault in an elastic medium, adjacent to or overlying a velocity strengthening (VS; creeping) region can rupture in earthquakes of variable magnitude, and exhibit temporal clustering, if it exceeds a critical dimension relative to the nucleation size.

For 2-D earthquake cycle simulations on homogeneous vertical, antiplane rate-state faults loaded by downdip creep (Supplementary Information, section 1), the transition is illustrated in Fig. 1 and was first observed by Werner and Rubin (2013). The smallest fault $\left(W=3.2 L_{\infty}\right.$, with $L_{\infty}$ the nucleation half-length defined below, associated with the transition from stable to unstable sliding) exhibits simple cycles of system-size events, while two earthquakes per cycle occur at $W=13 L_{\infty}$ and about 12 earthquakes per cycle at $W=415 L_{\infty}$ (where a "cycle" is the time between two full ruptures). Small faults are characteristic and periodic (Fig. 1a,c), and 2 rupture cycles have a bimodal distribution of rupture length and inter-event times (Fig. 1b,d). In contrast, earthquakes on the largest fault span two orders of magnitudes in rupture length and exhibit temporal clustering (Fig. 1e,f). Below I present a simple theoretical model for these results, 

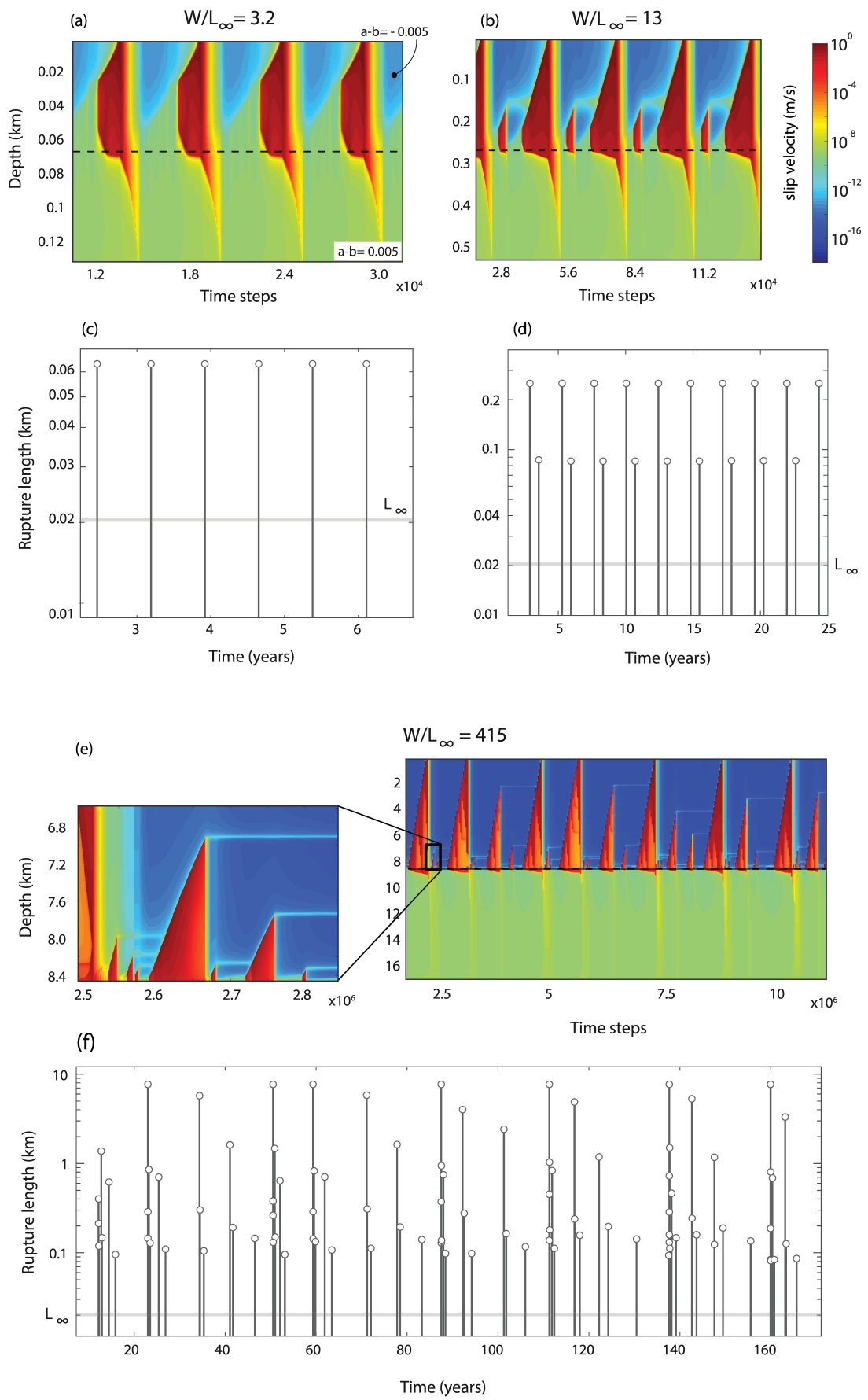

Figure 1. Examples of simulated cycles on antiplane vertical faults with variable $W / L_{\infty}$, with color indicating slip speed on a log scale. The dotted line marks the velocity-weakening to velocity-strengthening transition. The x-axis shows computational time steps. (a,c) For $W / L_{\infty}=3.2$ periodic full ruptures occur; (b,d) $W / L_{\infty}=13$ results in two ruptures per cycle; (e,f) $W / L_{\infty}=415$ presents a wider range of rupture dimensions and temporal clustering. The parameters for this simulation are $b=0.02, a-b= \pm 0.005$ in the VS/VW regions, $\sigma=50$ $\mathrm{MPa}, d_{c}=10^{-4} \mathrm{~m}$. Simulations are performed with the pseudo-dynamic code FDRA (Segall \& Bradley, 2012). 
compare them with empirical power-law distributions observed in nature, and discuss the implications for earthquake cycle models and seismic hazard.

\section{Theory}

Consider a stick-slip fault segment loaded by creep from an adjacent creeping region: for example, an isolated small asperity embedded in a creeping fault, or a long antiplane fault loaded by downdip creep. The stress state in the seismic area is determined by the slip that has taken place in the creeping region during the interseismic period since the last full rupture, $S(t)$. I argue that the seismic behavior is controlled by the ratio of interseismic slip required to nucleate an event $\left(S_{n}\right)$ to the slip required for a systemsize event $\left(S_{\text {full }}\right)$. Intuitively, we may expect $S_{n}$ to increase with the nucleation dimension $L_{\infty}$, and the slip required for a full rupture $S_{f u l l}$ to increase with the size of the seismic region. Since $S_{n}$ is independent of $W$ and $S_{\text {full }}$ increases with $W$, we expect two regimes: for $S_{n} \geq S_{f u l l}$, all events are full ruptures, with simple characteristic cycles. For $S_{n}<S_{\text {full }}$, partial ruptures occur. The ratio of partial ruptures to full ruptures increases with $S_{\text {full }} / S_{n}$. From dimensional arguments (and confirmed more rigorously below), we may expect $S_{f u l l} / S_{n}$ to be an increasing function of $W / L_{\infty}$ : this ratio defines the different regimes and degree to which the fault is characteristic.

To make this argument quantitative, the problem can be described as follows. A seismic segment of extent $W$, characterized by velocity weakening (VW) friction, starts slipping where the stress is highest: at the boundary with the creeping region, characterized by velocity strengthening (VS) friction. Such slip is described as a 1D crack in an infinite medium loaded by end-point displacement $S(t)$ applied at the VW-VS transition, from which slip penetrates into the VW region (see Fig. 1(a,b,e)). Quasi-static crack propagation is controlled by an energy balance criterion (Griffith, 1921), equivalent to requiring the stress intensity factor (SIF) $K$ at the crack tip to be equal to the fracture toughness $K_{c}$ (Irwin, 1957).

The stress intensity factor can be written as $K=K_{l}-K_{\Delta \tau}$, where $K_{l}$ is the SIF due to loading a stress free crack, and $K_{\Delta \tau}$ accounts for uniform stress changes within the crack. The quasi-static equation of motion for the crack tip at distance $l$ from the load point is given by:

$$
K_{l}(l)-K_{\Delta \tau}(l)=K_{c} .
$$


After each earthquake, the stress near the VS-VW transition is low and the fault is locked; the loading term $K_{l}(l)$ increases with time as slip accumulates in the VS region, and creep penetrates within the VW region. When it reaches a critical distance $L_{n}$, nucleation occurs. During creep propagation, the fracture energy term is typically negligible (Cattania \& Segall, 2019), so eq. 1 can be written as $K_{l}(l) \approx K_{\Delta \tau}(l)$. The stress intensity factors are $K_{l}=\mu^{\prime} S / \sqrt{2 \pi l}$ and $K_{\Delta \tau}=\Delta \tau \sqrt{\pi l / 2}$ (Tada et al., 2000), where $\mu^{\prime}=\mu$ for antiplane and $\mu^{\prime}=\mu /(1-\nu)$ for plane strain deformation $(\mu$ is the shear modulus, $\nu$ the Poisson ratio); $\Delta \tau$ is the stress increase behind the creep front, which is equal and opposite to the stress drop in the previous event (Supplementary Information, section 3). Therefore the displacement required for the creep front to penetrate a distance $L_{n}$ is:

$$
S_{n}=\frac{\pi \Delta \tau}{\mu^{\prime}} L_{n}
$$

As proposed by Hawthorne and Rubin (2013), eq. 1 can also be used to estimate the minimum time between full ruptures. The condition for full rupture can be simplified noticing that $K_{\Delta \tau}=0$ over an entire cycle (Cattania \& Segall, 2019; Werner \& Rubin, 2013), so that $K_{l}=K_{c}$. With the expression for $K_{l}$ given above, the SIF is minimum at the top of the fault $(l=W)$. Therefore the displacement required for a full rupture is:

$$
S_{f u l l}=\frac{\sqrt{2 \pi W} K_{c}}{\mu^{\prime}}
$$

These critical displacements can be used to estimate the relative number of earthquake nucleations and full ruptures. Suppose that slip in the creeping region accumulates at a rate $\dot{S}=V_{p l}$ (averaged across a cycle). The average seismicity rate and the rate of full ruptures are simply $r=\dot{S} / S_{n}$ and $r_{f u l l}=\dot{S} / S_{\text {full }}$, and the total number of earthquakes per cycle is

$$
\alpha=\frac{r}{r_{f u l l}}=\frac{S_{f u l l}}{S_{n}} \sim \frac{K_{c} \sqrt{W}}{\Delta \tau L_{n}}
$$

Partial ruptures occur when $\alpha>1$ : the critical slip required for nucleation is smaller than the slip for a full rupture. This condition can be expressed in terms of the ratio $W / L_{\text {crit }}$ with $L_{c r i t} \sim\left(L_{n} \Delta \tau / K_{c}\right)^{2}$. Note that the quantities $L_{n}, K_{c}$ and $\Delta \tau$ are determined by the elastic and frictional properties of the fault, and do not depend on any actual, measurable length scale. For certain frictional laws, this expression can be simplified by considering how $L_{n}$ depends on $K_{c}$ and $\Delta \tau$ : as shown in the Supplementary Information, 
section 2 , for rate-state ageing law simulations $L_{c r i t}$ is proportional to the nucleation length $L_{\infty}$ derived by Rubin and Ampuero (2005):

$$
L_{\infty}=\frac{b}{\pi(b-a)^{2}} \frac{\mu^{\prime} d_{c}}{\sigma},
$$

where $\sigma$ is the normal stress and $a, b$ and $d_{c}$ are rate-state parameters. In this case, the number of earthquakes per cycle is

$$
\alpha \sim \sqrt{\frac{W}{L_{\infty}}} .
$$

The ratio $W / L_{\infty}$ determines the seismic regime of the fault: characteristic and periodic if $\alpha \leq 1$, bimodal for $2 \geq \alpha>1$, and with a vanishingly small fraction of systemsize ruptures as $\alpha \gg 1$. While this result was derived for cracks in a 2-D medium, the same transitions are expected to occur for other geometries. The 2-D result for the nucleation criterion (eq. 2) is also the limit for a circular asperity with $R \gg L_{n}$, or indeed any geometry with a local curvature radius $\gg L_{n}$. The scaling of the critical slip required for a full rupture (eq. 3) is also common to other geometries, and varies only by a geometrical factor of order 1, as demonstrated by Cattania and Segall (2019) for circular asperities and in the Supplementary Information, section 3 for a vertical fault reaching the free surface.

I test these predictions against numerical simulations of vertical antiplane faults (Fig. 1), described in the Supplementary Information (section 1). For this simple geometry, the only modification to the theory above consists of including the effect of the free surface, which modifies eq. 3 by a factor $\phi=0.71$ (Supplementary Information, section 3). Using expressions for fracture energy from rate-state friction the condition $\alpha=$ 1 is satisfied by

$$
\alpha \approx 0.45 \sqrt{\frac{W}{L_{\infty}}} .
$$

Therefore partial ruptures are possible when $\alpha>1$ or $W>(5 \pm 2) L_{\infty}$ (the range corresponds to the standard deviation of $L_{n} / L_{\infty}$, as shown in the Supplementary Information, Fig. 2). Fig. 2a shows a set of simulations with variable frictional (rate-state) parameters and fault dimension: $W / L_{\infty}=5 \pm 2$ is a reasonable approximation of the transition between single to double rupture cycles. The number of earthquakes per cycle is also well fit, to first order, by eq. 7 (Fig. 2b). Note that eq. 7 is derived assuming that each displacement increment $S_{n}$ corresponds to a single rupture; as discussed in the 
(a)

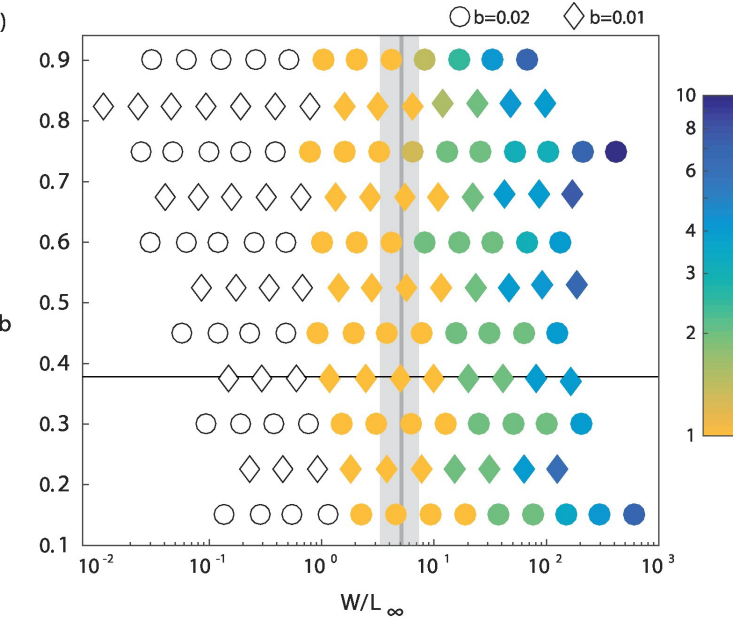

(b)

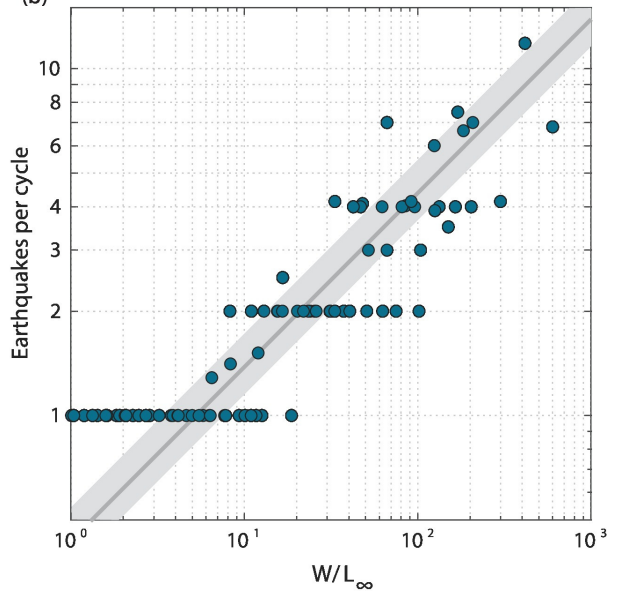

Figure 2. (a) number of earthquakes per cycle with variable $W / L_{\infty}$ and rate-state parameters $a, b$. The transition occurs near the value of $W / L_{\infty}$ predicted by the crack model and shown by the grey line, indicating the estimated value for $L_{n}=(2.9 \pm 0.6) L_{\infty}$ (Supplementary Figure S2). Symbols refer to two values of rate-state parameters $b$ (0.01, diamonds; 0.02 , circles), and white symbols on the left represent aseismic simulations. The horizontal line marks the value of $a / b$ above which the nucleation length is given by $L_{\infty}$ (Rubin \& Ampuero, 2005) and the scaling is expected to apply. (b) number of ruptures per cycle vs. $W / L_{\infty}$, compared with eq. 7 (grey line). Fractional values in the simulations are caused by the alternation of cycles with a different number of events. 

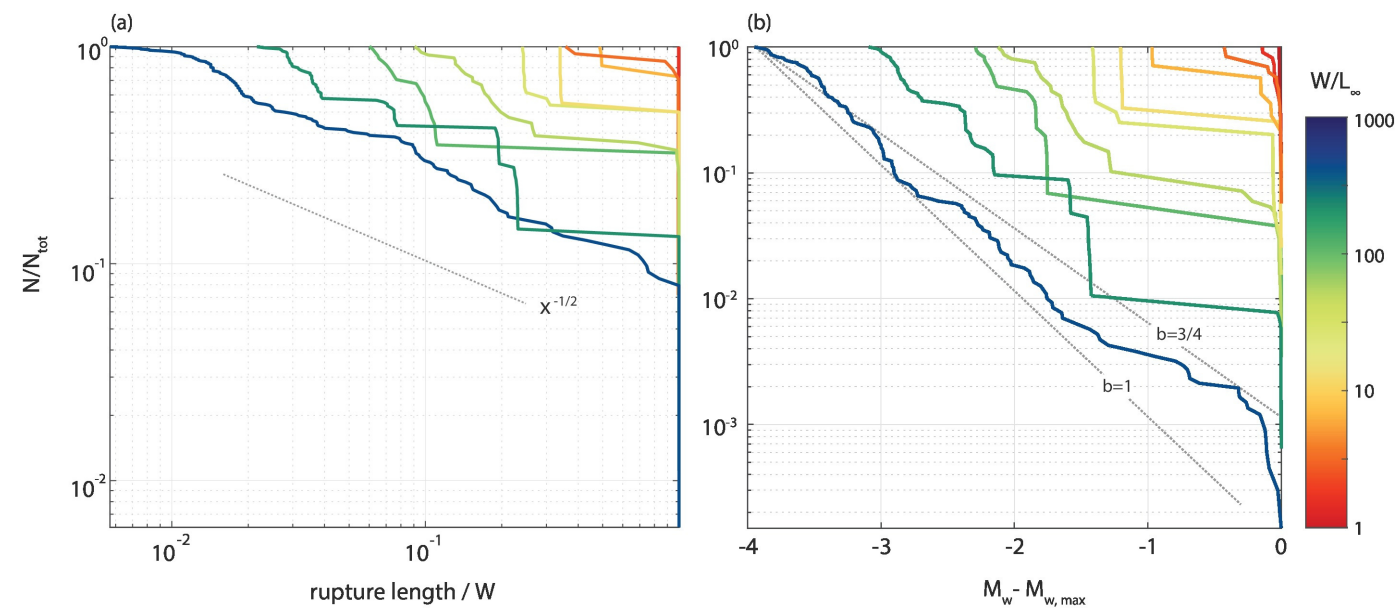

Figure 3. (a) Distribution of rupture lengths for simulations with $a / b=0.75$ showing characteristic distribution at small $W / L_{\infty}$; a bimodal distribution for $W / L_{\infty} \sim 10$; a truncated power-law distribution for large $W / L_{\infty}$ (up to 415). $N / N_{\text {tot }}$ is the normalized survival function (fraction of events at least equal to a certain rupture length). The dotted line shows the the power-law exponent consistent with the scaling in eq. 6. (b) Frequency-magnitude distribution from ruptures in a 3-D medium estimated as outlined in section 4 of the Supplementary Information. The dotted lines show a GR b-value of 0.75 and 1.0.

next section, this may not be true in 3-D, and the actual number of events per cycle can be higher than this (and may be estimated by geometrical arguments, see Supplementary Information, section 4). Moreover, in addition to the interseismic displacement accrued in the creeping region, the stress field is modified by the occurrence of partial ruptures: for example, the area of lower stress that can stop propagation of a further rupture (e.g. Lapusta (2003)). I find that this can lead to the occurrence of partial ruptures even when the energy criterion above is satisfied $\left(S(t)>S_{f u l l}\right)$, and increase the fraction of partial ruptures relative to eq. 7 .

\section{Distribution of rupture lengths and magnitudes}

The distribution of rupture lengths is characteristic for small seismogenic regions and bimodal for those large enough to have a partial rupture per cycle (Fig. 3). If the seismogenic region exceeds hundreds of nucleation lengths, and multiple (>10) ruptures per cycle, the distribution appears close to a power-law truncated at the characteristic length $W$, and spans two orders of magnitude. I do not attempt to derive this distribu- 
tion from first principles, but instead seek the power-law exponent consistent with previous results. Consider a survival function of the form $N(l)=A l^{-\gamma}$ between $L_{\text {min }}$ and $L_{\max }$, where $A$ is a constant and $N$ is the number of events with rupture length $\geq l$. The total number of events is simply $A L_{m i n}^{-\gamma}$, and the number of events with characteristic length $L_{\max }$ is $A L_{\max }^{-\gamma}$ (there are $A L_{\max }^{-\gamma}$ events greater than or equal to $L_{\max }$, and zero events greater than or equal to $\left(L_{\max }\right)+\varepsilon$, with $\varepsilon$ an arbitrarily small positive number, since the distribution is truncated). Therefore the number of events per cycle is $\left(L_{\min } / L_{\max }\right)^{-\gamma}$. Setting $L_{\min } \sim L_{\infty}$ and $L_{\max }=W$, and comparing this result with eq. 6 , implies $\gamma=$ $1 / 2$, which is indeed the value in the simulations (Fig. 3(a)).

Estimating the frequency-magnitude distribution for a 2-D fault embedded in a 3D medium requires some assumptions on the rupture length along strike, and the number of ruptures corresponding to each event simulated in 2-D. Assuming that all ruptures have the same aspect ratio and stress drop, the survival function in terms of seismic moments has the form: $N\left(M_{0}\right) \sim M_{0}^{-1 / 2}$, corresponding to a Gutenberg-Richter $b$-value of 3/4 (Supplementary Information, section 4). Fig. 3(b) shows the frequency-magnitude distribution obtained after weighting each simulated event by the number of equivalent ruptures in $3-\mathrm{D}$, assuming constant aspect ratio. The $b$-value is close to the theoretical value of $3 / 4$, but slightly larger; this seems to be related to a weakly sublinear scaling of slip with rupture length, implying a weak decrease in stress drop. The exponent is remarkably close to the typical $b$-value of 1 . However, the assumptions made when converting ruptures simulated in 2-D into equivalent $3-\mathrm{D}$ ones should be verified by running simulations in 3-D, and considering rupture propagation along strike after reaching the free surface.

\section{Inter-event time distributions}

The distribution of inter-event times also undergoes a similar transition from peaked at low $W / L_{\infty}$, to power-law at high $W / L_{\infty}$ (Fig. 4(a-b)). The coefficient of variation in inter-event time is $\sim 10^{-4}-10^{-3}$ for $W / L_{\infty}<4$, indicating almost perfectly periodic behavior; it increases to 1.5 for the largest $W / L_{\infty}$ (415), indicating clustering. I compare the inter-event time distributions with those typically found in seismic catalogs: Fig. 4(c) shows the distribution of inter-event times, normalized by the average rate, for $M_{w} \geq$ 6.0 events in the ANSS (Advanced National Seismic System) Comprehensive Earthquake Catalog. Corral (2004) first noted that the inter-event time distribution rescaled by the 

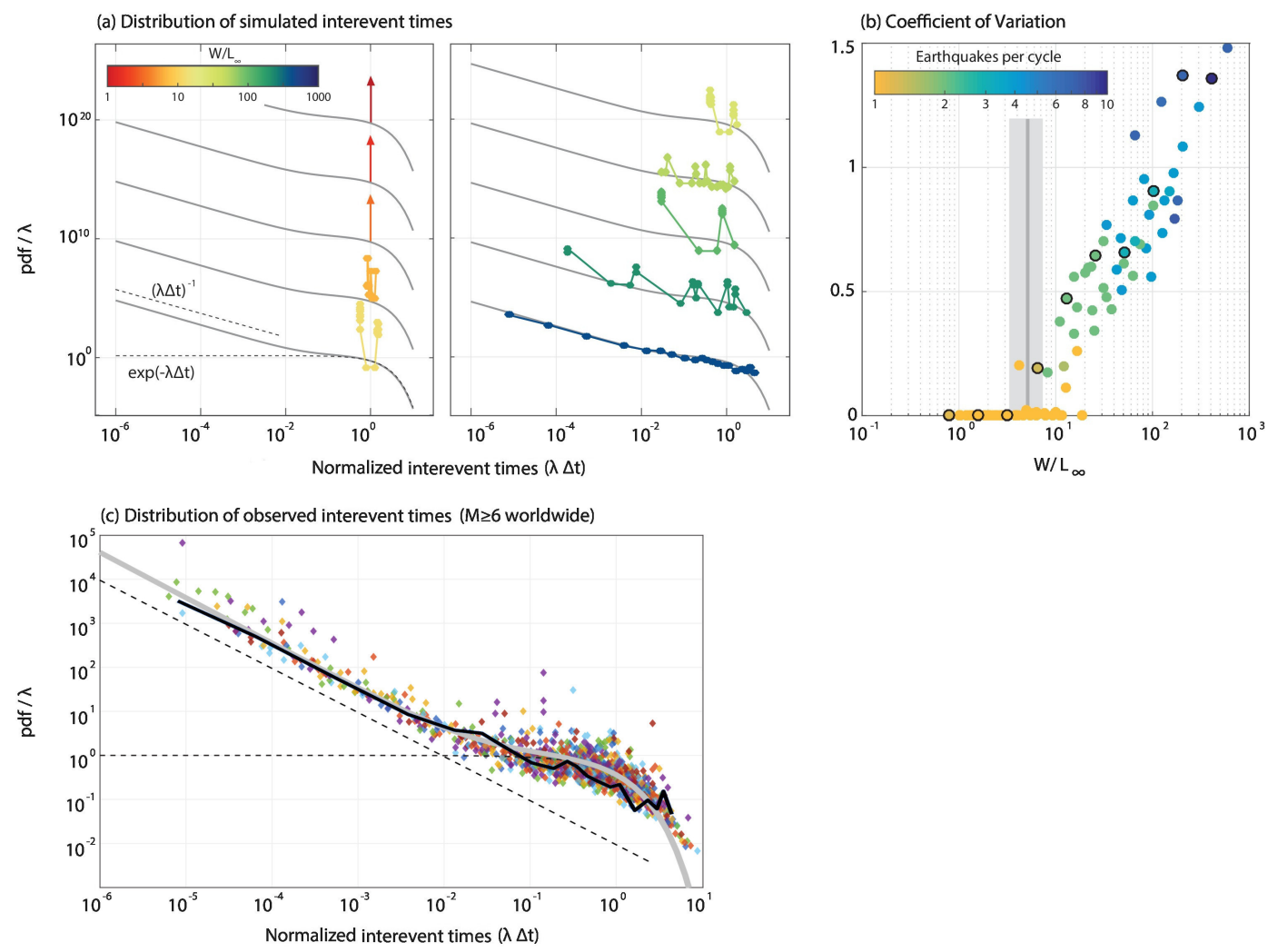

Figure 4. (a) Probability density function of normalized inter-event times calculated between any two consecutive events (full or partial ruptures) for the simulations with $a / b=0.75$. The grey lines are expressions for the universal scaling observed in nature (Corral (2004); Saichev and Sornette (2007); eq.16 in the Supplementary Information) compared with a $1 / t$ decay and the exponential distribution typical of Omori decay and Poisson sequences respectively (black dotted lines). Simulations with a coefficient of variation smaller than $10^{-3}$ are indicated by an arrow (delta function). Each curve is offset by $10^{5}$ for clarity. (b) coefficient of variation (standard deviation in $\Delta t$ divided by the mean) as a function of $W / L_{\infty}$, color coded by number of events per cycle. Circled simulations are those shown in the left panel. (c) Distribution of normalized inter-event times for $M_{w} \quad \geq \quad 6$ earthquakes in the ANSS Comprehensive Earthquake Catalog (ComCat), 1980-2018. The catalog was subdivided into 60 regions of $2000 \mathrm{~km}$ x $2000 \mathrm{~km}$, shown by the different colors. Grey and dashed lines as in panel (a); the black line is the distribution for $W / L_{\infty}=415$, also shown in dark blue in panel (a). 
total rate $\lambda$ follows a universal form independent of location and selection criteria; later, Hainzl et al. (2006) and Saichev and Sornette (2007) demonstrated that this behavior is well described by combining short term Omori clustering and background Poissonian seismicity. This is described by a function given by eq.16 in the Supplementary Information (section 5) and shown by the grey lines in Fig. $4(\mathrm{a}, \mathrm{c})$. For $W / L_{\infty} \gg 1$, the simulated inter-event time distribution approaches this expression, with a clear power-law decay at short $\lambda \Delta t$, consistent with $1 / t$ Omori decay, and a slower decay at $\lambda \Delta t \gtrsim 0.1$. As shown in Fig. 4(c), the distribution at $W / L_{\infty}=415$ is consistent with the theoretical expression and observed seismicity. This indicates that stress interaction between faults and faults segments, while undoubtedly an important process for regional seismicity, is not required to produce the universal inter-event time distribution documented by Corral (2004).

Temporal clustering is a direct consequence of afterslip: at a constant creep rate, it would always take the same time to accumulate the displacement required for nucleation $S_{n}$, and inter-event times would be constant even for multi-rupture cycles. The increase in clustering on larger faults is caused by faster afterslip, caused by a wider afterslip region for larger ruptures. In the Supplementary Information (section 6) I use a simple spring-slider model introduced by Perfettini (2004) to estimate the postseismic creep rate at the loading point $S(t)$. This yields the following expression for the time to the first nucleation after a full rupture:

$$
T_{n}=\frac{\sigma(a-b)_{v s} L_{p}}{\mu^{\prime} V_{p l}} \log \left[\left(\frac{V_{c o}}{V_{p l}}\right)^{\pi \frac{L_{n}}{L_{p}}-1}+1\right],
$$

where $(a-b)$ are rate-state parameters in the VS region, $V_{c o}$ is the seismic slip speed and $L_{p}$ is the penetration distance of a rupture into the VS region, proportional to $W$. The duration of a cycle can be estimated from the amount of slip required for a full rupture: since creep rate averaged across a cycle is simply $V_{p l}$, one can write

$$
T_{\text {full }}=\phi S_{\text {full }} / V_{p l}
$$

with $S_{\text {full }}$ given by eq. 3 and $\phi=0.71$ a factor accounting for the free surface (Supplementary Information, section 3). As shown in Supplementary Figure 4, this expression is a lower bound to the occurrence of full ruptures in the simulations, and the time to the first nucleation is well approximated by eq. 8 . The strong temporal clustering for $W / L_{\infty}$ is due to the fact that the nucleation timescale (eq. 8) and the full rupture timescale (eq. 9) differ by more than 6 orders of magnitude for large $W / L_{\infty}$. 


\section{Implications for seismic hazard}

In this study I explore the statistical properties of seismic sequences on a fault adjacent to creep. I show that: 1. larger faults are intrinsically less characteristic, with the fraction of system size ruptures decreasing as $\sqrt{L_{c r i t} / W} ; 2$. for sufficiently large $W / L_{\text {crit }}$, the frequency-size distribution approaches a truncated power-law, with a theoretical GutenbergRichter $b$ value of $3 / 4 ; 3$. inter-event times display Omori type clustering driven by afterslip.

On a fundamental level, the occurrence of partial ruptures is due to gradients in the stress field, a phenomenon also reported in laboratory experiments (Kammer et al., 2015). In the present study, the $1 / x$ decay of stress from a dislocation represents the slip accumulated in the VS region (which results in the stress intensity factor $K_{l} \sim 1 / \sqrt{x}$ ). The observation that the stress concentration ahead of a propagating rupture is large enough to make the rupture unstoppable (Rice, 1993; Ben-Zion, 2008) is valid if the fault is relatively small, so that the difference between the stress at the nucleation point and the minimum stress along the rupture path is smaller. At the bottom of the seismogenic zone, $a-b$ can be close to 0 and the nucleation length may be rather large (of the order of $0.1-1 \mathrm{~km}$ for the ageing law with $d_{c} \sim 0.1 \mathrm{~m} \sigma \sim 10 \mathrm{MPa}$ ). This would result in $W / L_{\infty} \lesssim 100$ and few ruptures per cycle, as found by Lapusta (2003) for ageing law simulations with $d_{c}=0.14 \mathrm{~mm}$. On the other hand, the nucleation length in nature may be smaller than typically assumed in numerical studies due to smaller values of $d_{c}$ (close to typical laboratory values of $0.001-0.01 \mathrm{~mm}$, see Dieterich (1979); Marone (1998)). This would promote larger $W / L_{\infty}$, leading to more events per cycle and the power law distributions emerging at $W / L_{\infty} \gtrsim 10^{2}-10^{3}$. For other frictional weakening mechanisms, the ratio $W / L_{\text {crit }}$ may be different. For example, the slip law tends to result in smaller nucleation lengths (Ampuero \& Rubin, 2008), favoring partial ruptures; but the smaller fracture energy would instead promote full ruptures. Dynamic weakening also modifies the fracture energy. Viesca and Garagash (2015) derived expressions for the fracture energy due to thermal pressurization, and I verified that the recurrence interval of full ruptures and its scaling with $W$ can be estimated from the argument above (Cattania \& Segall, 2016), with their expression for fracture energy. It should be noted that for slipweakening processes with an increase of fracture energy with slip, the scaling between $S_{\text {full }}$ and $W$ will be different from what is derived here for constant $K_{c}$ (eq. 3). 
The results presented here are in agreement with, and generalize, previous numerical studies. Lapusta et al. (2000) first showed that decreasing the value of $d_{c}$ leads to the appearance of partial ruptures, and their number decreases slowly with $d_{c}$; this was later confirmed by Werner and Rubin (2013). Recent studies found an increase in partial ruptures and complexity with fault dimension in subduction zones (Herrendorfer et al., 2015) and 2-D antiplane faults loaded from both sides (Wu \& Chen, 2014; Erickson et al., 2011), confirming that the results of the present study apply also to other fault geometries and frictional laws.

There is ample evidence for partial ruptures in nature. On megathrust faults, moderate $\left(M_{w}>7\right)$ events have been observed in the downdip portion of the fault, in areas where large $\left(M_{w} 8-9\right)$ events have previously occurred; recent examples include the $2015 M_{w} 7.8$ Gorkha, Nepal (Avouac et al., 2015; Galetzka et al., 2015), and the 2016 $M_{w}$ 7.6 Melinka, Chile (Melgar et al., 2017) earthquake. Other events, including the 2014 $M_{w} 8.2$ Iquique, Chile earthquake (Schurr et al., 2014) ruptures a fraction of a locked patch, arresting along strike where earlier ruptures propagated. Partial ruptures have been attributed to geometrical or frictional fault heterogeneity (Qiu et al., 2016; Moreno et al., 2018; Li et al., 2018; Dal Zilio et al., 2019). Other studies invoke, as I do here, gradients in the stress field due to nonuniform loading (Herrendorfer et al., 2015; Michel et al., 2017), implying that partial ruptures may be rather common for sufficiently large velocity weakening regions. It is worth noting that computational limitations often require to impose unrealistically large values for some of the relevant lengthscales, for example by setting $d_{c}$ several orders of magnitude larger than experimental values. This is effectively equivalent to modeling a much smaller fault (low $W / L_{\text {crit }}$ ), thus requiring additional complexity to reproduce observed sequences.

To summarize, I show that simple, isolated faults do not necessarily produce limit cycles of characteristic and periodic ruptures. Power-law distributions commonly observed in nature (Gutenberg-Richter distribution and Omori decay) can occur on a planar, homogeneous fault as long as the ratio of its size to a critical dimension related to the nucleation length is large $\left(10^{2}-10^{3}\right)$. Natural faults additionally present geometrical and frictional heterogeneity, which can give rise to even more variability in rupture lengths and more complex temporal patterns; however, such heterogeneity is not required to arrest a rupture and produce complex seismic sequences. The fraction of characteristic ruptures is a decreasing function of $W / L_{\infty}$, with faults much larger than the nucleation length 
increasingly less characteristic. Simple energy arguments, which can be refined by considering the particular geometry of interest, can provide insight into the statistics of earthquakes on a fault and its seismic hazard.

\section{Acknowledgments}

I am grateful to Paul Segall and Sebastian Hainzl for discussions and guidance during this work, and to Allan Rubin for insightful comments. I thank the editor Gavin Hayes, Chris Scholz and an anonimous reviewer for constructive reviews that improved the quality of the paper. The ANSS Comprehensive Earthquake Catalog can be downloaded at: https://earthquake.usgs.gov/data/comcat/. C.C. was supported by the German Academic Exchange Service (DAAD) with funds from the German Federal Ministry of Education and Research (BMBF) and the People Programme (Marie Curie Actions) of the European Union's Seventh Framework Programme (FP7/2007-2013) under REA grant agreement no. 605728, NSF award no. 1620496, and SCEC award no. 17144.

\section{References}

Ampuero, J., \& Rubin, A. (2008). Earthquake nucleation on rate and state faults-Aging and slip laws. Journal of Geophysical Research: Solid Earth, 113(August 2007), 1-61. doi: 10.1029/2007JB005082

Aochi, H., \& Ide, S. (2009). Complexity in earthquake sequences controlled by multiscale heterogeneity in fault fracture energy. Journal of Geophysical Research, 114(B3), B03305. doi: 10.1029/2008JB006034

Avouac, J.-P., Meng, L., Wei, S., Wang, T., \& Ampuero, J.-P. (2015). Lower edge of locked Main Himalayan Thrust unzipped by the 2015 Gorkha earthquake. Nature Geoscience, 8(9), 708-711. doi: 10.1038/ngeo2518

Bak, P., \& Tang, C. (1989). Earthquakes as a self-organized critical phenomenon. Journal of Geophysical Research: Solid Earth, 94(B11), 15635-15637. doi: 10 $.1029 / J B 094$ iB11p15635

Ben-Zion, Y. (2008). Collective behavior of earthquakes and faults: Continuumdiscrete transitions, progressive evolutionary changes, and different dynamic regimes. Reviews of Geophysics, 46(4), RG4006. doi: 10.1029/2008RG000260

Ben-Zion, Y., \& Rice, J. R. (1995). Slip patterns and earthquake populations along different classes of faults in elastic solids. Journal of Geophysical Research: 
Solid Earth, 100(B7), 12959-12983. doi: 10.1029/94JB03037

Burridge, R., \& Knopoff, L. (1967). Model and theoretical seismicity. Bulletin of the Seismological Society of America, 57(3), 341-371.

Cattania, C., \& Segall, P. (2016). Earthquake cycles on rate-state faults: How does recurrence interval and its variability depend on fault length? Poster Presentation at 2016 SCEC Annual Meeting., 2016.

Cattania, C., \& Segall, P. (2019). Crack models of repeating earthquakes predict observed moment-recurrence scaling. Journal of Geophysical Research: Solid Earth, 124(1), 476-503. doi: 10.1029/2018JB016056

Corral, Á. (2004). Long-term clustering, scaling, and universality in the temporal occurrence of earthquakes. Physical Review Letters, 92(10), 108501. doi: 10 .1103/PhysRevLett.92.108501

Dal Zilio, L., van Dinther, Y., Gerya, T., \& Avouac, J.-P. (2019). Bimodal seismicity in the Himalaya controlled by fault friction and geometry. Nature Communications, 10(1), 48. doi: 10.1038/s41467-018-07874-8

Dieterich, J. H. (1979). Modeling of Rock Friction Experimental Results and Constitutive Equations. Journal Geophys. Res., 84(9), 2161-2168.

Dublanchet, P., Bernard, P., \& Favreau, P. (2013). Interactions and triggering in a 3-D rate-and-state asperity model. Journal Geophys. Res., 118(April), 22252245. doi: 10.1002/jgrb.50187

Erickson, B. A., Birnir, B., \& Lavallée, D. (2011). Periodicity, chaos and localization in a Burridge-Knopoff model of an earthquake with rate-and-

state friction. Geophysical Journal International, 187(1), 178-198. $\quad$ doi: 10.1111/j.1365-246X.2011.05123.x

Galetzka, J., Melgar, D., Genrich, J. F., Geng, J., Owen, S., Lindsey, E. O., .. Maharjan, N. (2015). Slip pulse and resonance of the Kathmandu basin during the 2015 Gorkha earthquake, Nepal. Science, $349(6252), 1091-1095$. doi: $10.1126 /$ science.aac6383

Griffith, A. A. (1921). The phenomena of rupture and flow in solids. Philosophical Transactions of the Royal Society A: Mathematical, Physical and Engineering Sciences, 221 (582-593), 163-198. doi: 10.1098/rsta.1921.0006

Hainzl, S., Scherbaum, F., \& Beauval, C. (2006). Estimating background activity based on interevent-time distribution. Bulletin of the Seismological Society of 
America, 96(1), 313-320. doi: 10.1785/0120050053

Hawthorne, J. C., \& Rubin, A. M. (2013). Laterally propagating slow slip events in a rate and state friction model with a velocity-weakening to velocitystrengthening transition. Journal of Geophysical Research: Solid Earth, 118(7), 3785-3808. doi: 10.1002/jgrb.50261

Herrendorfer, R., van Dinther, Y., Gerya, T., \& Dalguer, L. A. (2015). Earthquake supercycle in subduction zones controlled by the width of the seismogenic zone. Nature Geosci, 8(6), 471-474. doi: 10.1038/ngeo2427

Hillers, G., Mai, P. M., Ben-Zion, Y., \& Ampuero, J.-P. (2007). Statistical properties of seismicity of fault zones at different evolutionary stages. Geophysical Journal International, 169(2), 515-533. doi: 10.1111/j.1365-246X.2006.03275 . $\mathrm{x}$

Irwin, G. (1957). Analysis of stresses and strains near the end of a crack traversing a plate. Journal of Applied Mechanics, 24(Sep), 361-364. doi: noDOI

Kagan, Y. Y., Jackson, D. D., \& Geller, R. J. (2012). Characteristic Earthquake Model, 1884-2011, R.I.P. Seismological Research Letters, 83(6), 951-953. doi: $10.1785 / 0220120107$

Kammer, D. S., Radiguet, M., Ampuero, J. P., \& Molinari, J. F. (2015). Linear elastic fracture mechanics predicts the propagation distance of frictional slip. Tribology Letters, 57(3). doi: 10.1007/s11249-014-0451-8

Kaneko, Y., Avouac, J.-p., \& Lapusta, N. (2010). Towards inferring earthquake patterns from geodetic observations of interseismic coupling. Nature Geoscience, 3(5), 363-369. doi: 10.1038/ngeo843

Lapusta, N. (2003). Nucleation and early seismic propagation of small and large events in a crustal earthquake model. Journal of Geophysical Research, 108, 118. doi: 10.1029/2001JB000793

Lapusta, N., Rice, J. R., Ben-Zion, Y., \& Zheng, G. (2000). Elastodynamic analysis for slow tectonic loading with spontaneous rupture episodes on faults with rate- and state-dependent friction. Journal of Geophysical Research: Solid Earth, 105(B10). doi: 10.1029/2000JB900250

Li, S., Barnhart, W. D., \& Moreno, M. (2018). Geometrical and frictional effects on incomplete rupture and shallow slip deficit in ramp-flat structures. Geophysical Research Letters, 45(17), 8949-8957. doi: 10.1029/2018GL079185 
Marone, C. (1998). Laboratory-derived friction laws and their application to seismic faulting. Annual Review of Earth and Planetary Sciences, 26(1), 643-696. doi: 10.1146/annurev.earth.26.1.643

Melgar, D., Riquelme, S., Xu, X., Baez, J. C., Geng, J., \& Moreno, M. (2017). The first since 1960: A large event in the Valdivia segment of the Chilean Subduction Zone, the 2016 M7.6 Melinka earthquake. Earth and Planetary Science Letters, 474, 68-75. doi: 10.1016/j.epsl.2017.06.026

Michel, S., Avouac, J.-P., Lapusta, N., \& Jiang, J. (2017). Pulse-like partial ruptures and high-frequency radiation at creeping-locked transition during megathrust earthquakes. Geophysical Research Letters, 44 (16), 8345-8351. doi: 10.1002/2017GL074725

Moreno, M., Li, S., Melnick, D., Bedford, J. R., Baez, J. C., Motagh, M., ... Oncken, O. (2018). Chilean megathrust earthquake recurrence linked to frictional contrast at depth. $\quad$ Nature Geoscience, 11(4), $285 . \quad$ doi: $10.1038 /$ s41561-018-0089-5

Mulargia, F., Stark, P. B., \& Geller, R. J. (2017). Why is probabilistic seismic hazard analysis (PSHA) still used? Physics of the Earth and Planetary Interiors, 264, 63-75. doi: 10.1016/J.PEPI.2016.12.002

Olami, Z., Feder, H. J. S., \& Christensen, K. (1992). Self-organized criticality in a continuous, nonconservative cellular automaton modeling earthquakes. Physical Review Letters, 68(8), 1244-1247. doi: 10.1103/PhysRevLett.68.1244

Page, M. (2010). Reply to Schwartz and Open Discussion. Seismol. Res. Lett, 81, 331.

Page, M., \& Felzer, K. $\quad$ (2015). Southern San Andreas fault seismicity is consistent with the Gutenberg-Richter magnitude-frequency distribution.

Bulletin of the Seismological Society of America, 105(4), 2070-2080. doi: $10.1785 / 0120140340$

Parsons, T., Console, R., Falcone, G., Murru, M., \& Yamashina, K. Comparison of characteristic and Gutenberg - Richter models for timedependent $\mathrm{M} \geq 7.9$ earthquake probability in the Nankai-Tokai subduction zone, Japan. Geophysical Journal International, 190(3), 1673-1688. ～doi: 10.1111/j.1365-246X.2012.05595.x

Parsons, T., \& Geist, E. L. (2009). Is there a basis for preferring characteristic 
earthquakes over a gutenberg-richter distribution in probabilistic earthquake forecasting? Bulletin of the Seismological Society of America, 99(3), 20122019. doi: $10.1785 / 0120080069$

Parsons, T., Geist, E. L., Console, R., \& Carluccio, R. (2018). Characteristic earthquake magnitude frequency distributions on faults calculated from consensus data in California. Journal of Geophysical Research: Solid Earth, 123(12), 2018JB016539. doi: 10.1029/2018JB016539

Perfettini, H. (2004). Postseismic relaxation driven by brittle creep: A possible mechanism to reconcile geodetic measurements and the decay rate of aftershocks, application to the Chi-Chi earthquake, Taiwan. J. Geophys Res., 109(B2), B02304. doi: 10.1029/2003JB002488

Qiu, Q., Hill, E. M., Barbot, S., Hubbard, J., Feng, W., Lindsey, E. O., ... Tapponnier, P. (2016). The mechanism of partial rupture of a locked megathrust: The role of fault morphology. Geology, 44(10), 875-878. doi: 10.1130/G38178.1

Rice, J. R. (1993). Spatio-temporal complexity of slip on a fault. Journal of Geophysical Research, 98(B6), 9885. doi: 10.1029/93JB00191

Rubin, A. M., \& Ampuero, J. (2005). Earthquake nucleation on (aging) rate and state faults. Journal of Geophysical Research, 110(2), 1-24. doi: 10.1029/ 2005JB003686

Saichev, A., \& Sornette, D. (2007). Theory of earthquake recurrence times. Journal of Geophysical Research: Solid Earth, 112(4). doi: 10.1029/2006JB004536

Schurr, B., Asch, G., Hainzl, S., Bedford, J., Hoechner, A., Palo, M., ... Vilotte, J.-P. (2014). Gradual unlocking of plate boundary controlled initiation of the 2014 Iquique earthquake. Nature, 512(7514), 299-302. doi: $10.1038 /$ nature13681

Schwartz, D. P. (2010). Do large earthquakes on faults follow a Gutenberg-Richter or characteristic distribution?: A characteristic view. Seismol. Res. Lett, 81, 331.

Segall, P., \& Bradley, A. (2012). Slow-slip evolves into megathrust earthquakes in 2D numerical simulations. Geophysical Research Letters, 39(2-6), . doi: 10 $.1029 / 2012$ GL052811

Stirling, M., \& Gerstenberger, M. (2018). Applicability of the Gutenberg-Richter relation for major active faults in new zealand. Bulletin of the Seismological So- 
ciety of America, 108(2), 718-728. doi: 10.1785/0120160257

Tada, H., Paris, P. C., \& Irwin, G. R. (2000). The Stress Analysis of Cracks Handbook. Hellertown PA: Del Research Corp. doi: 10.1115/1.801535

Tse, S. T., \& Rice, J. R. (1986). Crustal earthquake instability in relation to the depth variation of frictional slip properties. Journal of Geophysical Research, 91 (B9), 9452. doi: 10.1029/JB091iB09p09452

Viesca, R. C., \& Garagash, D. I. (2015). Ubiquitous weakening of faults due to thermal pressurization. Nature Geosci, 8(October). doi: 10.1038/NGEO2554

Werner, M., \& Rubin, A. (2013). Mechanical erosion of the seismogenic zone by creep from below on rate-and-state faults. In AGU Fall Meeting Abstracts. San Francisco.

Wu, Y., \& Chen, X. (2014). The scale-dependent slip pattern for a uniform fault model obeying the rate- and state-dependent friction law. Journal of Geophysical Research: Solid Earth, 119(6), 4890-4906. doi: 10.1002/2013JB010779 